Archives

6 | 1990

Varia

\title{
La forêt : éléments du dossier dans l'inde
}

\section{Francis Zimmermann}

\section{(2) OpenEdition}

\section{Journals}

Édition électronique

URL : http://journals.openedition.org/ccrh/2863

DOI : $10.4000 /$ ccrh. 2863

ISSN : $1760-7906$

Éditeur

Centre de recherches historiques - EHESS

Édition imprimée

Date de publication : 15 octobre 1990

ISSN : 0990-9141

Référence électronique

Francis Zimmermann, "La forêt : éléments du dossier dans l'inde », Les Cahiers du Centre de Recherches Historiques [En ligne], 6| 1990, mis en ligne le 20 mars 2009, consulté le 10 décembre 2020. URL : http://journals.openedition.org/ccrh/2863 ; DOI : https://doi.org/10.4000/ccrh.2863

Ce document a été généré automatiquement le 10 décembre 2020.

Article L.111-1 du Code de la propriété intellectuelle. 


\title{
La forêt : éléments du dossier dans l'inde
}

\author{
Francis Zimmermann
}

Des mouvements d'agitation populaire visant à empêcher la mise en coupe des forêts dans l'Himalaya, comme le Chipko andolan, ont eu un certain retentissement hors des frontières de l'Inde, mais on ne dit pas assez que ces mouvements ont une histoire déjà vieille de plus d'un siècle. Les historiens et sociologues indiens de l'école des subaltern studies s'y sont intéressés et Ramachandra Guha, dans un livre récent en tous points admirable, a retracé l'histoire de la surexploitation des forêts depuis le siècle dernier et l'histoire des résistances populaires à cette déforestation. Nous nous bornerons ici à l'exposé de ces aspects contemporains du dossier « Forêt ».

2 S'il fallait être complet, nous devrions remonter au début de la période historique, quand l'Inde était encore couverte de forêts; nous devrions évoquer les premiers feux de brousse à l'Est du Dehli Doab, les premières trouées dans la forêt dense qui couvrait le cours moyen du Gange à l'âge de fer (1050-450 avant J.C.); nous devrions décrire les quatre grands types de forêts déterminés en Inde par le relief et la mousson: 1 - formations épineuses, 2 - forêt sèche à feuilles caduques, 3 - forêt humide à feuilles caduques et 4 - forêt sempervirente, s'étageant en gros d'Ouest en Est; la dialectique entre le village et la forêt, entre la plaine et la montagne. Tout ceci constitue une thématique féconde en histoire ancienne. Mais l'actualité nous invite à centrer cet exposé sur l'Inde moderne. Les chercheurs indiens eux-mêmes, aiguillonés par le débat politique sur la forêt et la protection de l'environnement, ont multiplié ces dernières années les enquêtes de terrain et les études d'histoire coloniale; essayons de poser quelques jalons dans une bibliographie en pleine expansion.

Deux remarques préliminaires s'imposent. On doit noter d'abord l'ampleur et la violence de la déforestation. On rappelera ensuite que, traditionnellement, la forêt indienne est une forêt habitée par l'homme. Les statistiques du Forest Department répètent depuis vingt ans que les forêts couvriraient environ $23 \%$ du territoire national, avec des pointes au Tripura ( $60 \%$ de la superficie totale de cette région), en Orisa ( $43 \%$ ), mais partout 
ailleurs une densité inférieure à $40 \%$. Les chiffres sont trompeurs. On veut dire en réalité que ces $23 \%$, c'est-à-dire 75 millions d'hectares, sont classés comme forest lands et placés sous le contrôle de l'administration des forêts. Mais y-a-t-il encore des arbres sur ces terres? Les enquêtes de terrain et les observations par satellite conduisent à penser que les forêts ne couvriraient aujourd'hui guère plus de 12 \% de la surface totale de l'Inde. La déforestation, selon les estimations de la FAO, faisait disparaître 155.000 hectares de forêts au début des années 1980. Les estimations actuelles multiplient ce chiffre par dix. L'Inde subirait une perte annuelle d'un million et demi d'hectares de forêts, se plaçant au deuxième rang mondial pour la vitesse de déforestation, entre le Brésil (huit millions d'hectares par an) et l'Indonésie (900.000 hectares par an), dans un tableau qui ne tient pas compte encore de la nouvelle politique de mise en coupe des forêts pour renflouer les finances publiques en Birmanie ${ }^{1}$. Une autre façon d'apprécier la violence du processus est de voir son impact sur la flore, la faune et la santé publique. L'un des chapitres les plus dramatiques de l'histoire contemporaine de la déforestation est le massacre des antilopes qui suivit immédiatement la proclamation de l'Indépendance en $1947^{2}$. Autre histoire exemplaire qui prend tout son sens dans la perspective de l'anthropologie médicale, signalons l'étude déjà classique de Nichter ${ }^{3}$ sur la destruction de la forêt de Kyasanur il y a dix ans. Les tiques chassées de la forêt se sont rabattues sur l'espace rural, provoquant des épidémies de grippe et d'encéphalite mortelles que les habitants interprétèrent comme un châtiment infligé par les dieux de la forêt détruite.

4 A l'exception des zones inaccessibles dans l'Himalaya, les forêts de l'Inde ne sont pas absolument sauvages. Dans les forêts même les plus reculées, ou bien l'on trouve traditionellement des aborigènes ou bien l'administration coloniale a implanté de la main d'oeuvre en créant, à partir de l'Indian Forest Act de 1878, des forest villages ${ }^{4}$. Bien que la plupart des villages forestiers se soient fondus par la suite dans la catégorie plus générale des revenue villages, il en reste quelque 5.000 dans la catégorie juridique des forest villages, abritant environ 48 millions d'habitants qui vivent donc en forêt ou à l'orée de la forêt dont ils tirent leur subsistance ${ }^{5}$. Autrement dit, la forêt indienne est une forêt habitée.

5 La forêt habitée, aux confins du monde rural, est le lieu d'un conflit entre l'Etat colonial et la population locale depuis le milieu du siècle dernier. Je voudrais esquisser l'analyse de ce conflit sur deux plans, d'abord l'histoire coloniale puis la sociologie, le Chipko andolan ${ }^{6}$ est un point de départ commode, à partir duquel nous pouvons remonter dans le temps à la recherche de ses conditions d'émergence. Conditions historiques et conditions sociologiques. C'est un mouvement rural d'agitation non-violente né en mars 1973 dans l'Himalaya pour défendre les forêts contre l'abattage. Les femmes du village se mobilisaient pour barrer l'accès des bûcherons aux arbres de la forêt voisine; elles s'enchaînaient aux arbres, les étreignaient, les embrassaient. Les intellectuels engagés ont aussitôt rejoint ce mouvement écologiste né at the grass roots, qui fit école ailleurs, en particulier au Karnataka. Cependant, l'analyse de Ramachandra Guha dont je vais m'inspirer est solidement enracinée dans un terroir, un cadre régional bien délimité par l'histoire et la sociologie, ce qui lui permet de mettre en évidence le lien traditionnel entre la forêt, les mouvements de résistance populaire et les institutions de la royauté. 


\section{Histoire de la mise en defens : l'accès des paysans à la forêt de 1864 a nos jours}

6 L'histoire des rapports entre les paysans, les autorités et la forêt dans l'Inde coloniale offre de nombreux points de comparaison avec ce qui s'est passé en Europe et en particulier en France, mais l'exploitation commerciale de la forêt et les rébellions paysannes qui s'ensuivirent sont nées un siècle plus tard que chez nous. Le tournant majeur, en Inde, est la construction d'un énorme réseau de voies ferrées, grandes consommatrices de traverses de sol (Shorea robusta), de teck, de cèdre déodar et, plus tard, de pin. Pour contrôler les coupes massives qu'entraîne l'expansion du réseau, les autorités britanniques créent en 1864 un Forest Department avec l'aide d'experts allemands. Une série de lois dont la plus importante est l'Indian Forest Act de 1878, réglementent l'accès des paysans à la forêt dite protégée et créent un domaine clos de forêts dites réservées. L'exploitation commerciale des futaies met directement l'Etat en conflit avec les paysans qui tiraient jusqu'alors de la forêt une grande partie de leurs ressources : le bois de chauffe et le bois d'oeuvre, le fourrage, des terres cultivables sur brûlis. Des règlements limitent ou interdisent désormais l'émondage, l'incendie et le pâturage en forêt ${ }^{7}$. C'est alors que naissent les premières rébellions qui constituent la préhistoire du mouvement Chipko; on enfreint les interdictions, on poursuit en secret la coutume des brûlis.

7 Je limiterai mes exemples à l'Himalaya, pour abréger, et donc à certains types de forêts. Mais ils valent pour toute l'Asie du Sud dans les régions où la foresterie et les plantations ont éliminé les traditions d'essartage et limité les droits d'usage. Par exemple, la dialectique du chêne et du pin que je vais évoquer d'après Guha se reproduit ailleurs sous d'autres formes: en particulier au Karnataka, sous la forme d'une dialectique entre le honge (pongamia glabra) ou le neem (azadirachta indica), espèces précieuses au paysan, et l'eucalyptus qu'on plante à des fins commerciales ${ }^{8}$. L'opposition des feuillus aux conifères, là où ils coexistent, illustre de façon frappante le conflit d'intérêts entre la population locale et les propriétaires de la forêt. Dans la forêt himalayenne entre $1.500 \mathrm{~m}$ et $2.000 \mathrm{~m}$ coexistent par exemple le banj (quercus incana) et le chir (pinus roxburghii), autrement dit, le chêne et le pin. Les chênes et autres feuillus sont les plus précieux pour l'agriculture locale, mais ils ne présentaient aucun intérêt aux yeux des autorités britanniques qui découvrent au contraire dans les dernières années du xix $x^{e}$ siècle que les pins constituent un précieux capital. C'est dans les années 1890 qu'on entreprit d'extraire du chir la résine de pin pour fabriquer la colophane et l'essence de térébenthine. De nouvelles méthodes de distillation permirent de décupler la production entre 1910 et 1920. Au même moment, les chimistes mirent au point un traitement de protection du bois permettant d'utiliser le chir dans les traverses de chemin de fer. Les droits des villageois sur les forêts du Kumaun et du Garhwal ont été progressivement restreints en raison inverse de cette expansion commerciale. Les autorités britanniques, sur la base du Forest Act de 1878, classèrent d'abord les forêts du Kumaun et du Garhawal dans le domaine réservé (reserved forest) ou dans le domaine protégé (protected forest) ; des coupes étaient concédées à des industriels pour les besoins de la sidérurgie. Mais en 1893, elles s'attaquèrent aux friches ${ }^{9}$ et toutes autres terres non arpentées, qui furent mises en défens et classées district protected forest, puis une partie de ces friches devinrent reserved 
forest en 1911. La coutume d'incendier le sous-bois pour créer des herbages fut interdite à moins d'un mille d'une forêt réservée, ce qui revenait pratiquement à la rendre illégale ${ }^{10}$.

La forêt de l'administrateur colonial est fort différente de celle du paysan. Le programme des forestiers dans l'Himalaya était de réduire les forêts mixtes de conifères et de feuillus à des peuplements homogènes, en abattant les chênes pour ne conserver que les pins ${ }^{11}$. La forêt du paysan est, au contraire, un milieu de vie diversifié où chaque espèce a ses usages. Le chêne donne du fourrage, de l'engrais (feuilles mélangées à la bouse), des litières, du bois de chauffage et des outils. Le pin fait d'excellentes torches, des charpentes; le tapis d'aiguilles sèches est incendié en avril-mai pour rendre la pente moins glissante tout en provoquant une repousse d'herbe luxuriante; là où l'herbe est rare, le pin lui-même donne du fourrage. D'autres exemples, d'autres terrains, d'autres espèces végétales permettraient de compléter cette typologie des forêts. Il y aurait la forêt du chasseur, celle de l'écobueur, etc. Voici seulement deux références pour éclairer l'exemple choisi sur deux points, l'histoire administrative et le régime foncier.

Les lois promulguées pour limiter les droits d'usage sur la forêt et l'administration centralisée qui entreprit de constituer un domaine clos à partir des années 1870, supposaient l'existence d'un cadastre. Les Anglais avaient, en effet, créé en 1818, le Great Trigonometral Survey of India, divisé en trois branches : la topographie, la longimétrie et le cadastre. Ce dernier, d'abord appelé revenue survey, fut rebaptisé Cadastral Survey et prit toute son importance sous l'impulsion du colonel Thuilier entre 1861 et 1878 ; c'est alors que les forêts commencèrent à être cadastrées. Faire l'histoire de la foresterie coloniale à travers celle des surveys (Cadastral, Botanical Survey, etc.), c'est étudier la dialectique du centre et de la périphérie, autrement dit, les conflits d'intérêt entre l'administration centrale et les hommes sur le terrain ${ }^{12}$.

Par ailleurs, il faut relativiser le cas des forêts du Kumaun et du Garhwal étudiées par Ramachandra Guha. Idéalisant la structure sociale, Guha croit voir s'épanouir dans les montagnes himalayennes une « communauté villageoise égalitaire $»^{13}$ qui échapperait à la triade Sarkar (l'État), Sahukar (l'usurier), Zamindar (le possesseur d'un droit de contrôle sur la terre) qui ailleurs formaient les trois piliers du pouvoir colonial. Mais cette peinture d'une Arcadie est peu convaincante. Le Sarkar est omniprésent, car la forêt est terre royale comme on va voir; forêts et friches n'échappent au régime des tenures foncières que dans la mesure où elles sont sous contrôle direct de l'Etat. Le Zamindar n'est pas absent non plus. Jean-Claude Galcy ${ }^{14}$ montre au contraire dans les tenures de cette région la coexistence de deux ayants droit sur une même parcelle, conjointement responsables devant l'impôt ; l'un possède un droit de contrôle et de faire-valoir indirect, l'autre, un fermage direct.

11 En réalité, l'histoire des forêts est indissociable de l'histoire de la propriété foncière. Dans la Présidence de Madras, par exemple, David Ludden ${ }^{15}$ rapporte que les autorités britanniques, en assignant les forêts au domaine public, provoquèrent une avalanche de procès où les Zamindars tentaient vainement de faire reconnaître leurs droits sur de vastes domaines boisés en zone montagneuse dont ils étaient ainsi dépossédés. Dans les hautes terres de la zone humide de Ccylan, Eric Meyer ${ }^{16}$ montre comment les plantations ont évincé les cultures sur brûlis et mordu sur la forêt ${ }^{17}$. A chaque fois, les forêts furent accaparées par l'Etat pour être concédées ou vendues à des exploitants commerciaux. 


\section{Les jacqueries, le roi et la forêt ; origines de l'écologie militante}

12 L'Indépendance n'a pas modifié l'idéologie dominante. Le gouvernement indien adopta en 1952 un National Forest Policy ou schéma directeur de son action sur la forêt qui prolongeait la politique britannique ${ }^{18}$ : les revendications locales ne devaient pas faire oublier « l'intérêt national » (\$7), à savoir, les besoins de la défense, des communications et des industries vitales (§ 13). Le mot d'ordre appliqué jusqu'à la fin des années 1970 était de développer les plantations pour produire du bois d'œuvre. Tel est le contexte dans lequel démarra le mouvement Chipko en 1973. Les premiers essais de foresterie sociale furent entrepris en 1977 et la nouvelle attitude des autorités, plus attentive aux besoins locaux (le bois de chauffe, le fourrage, la réhabilitation des friches), vient à peine d'être officialisée dans le National Forest Policy de $1988^{19}$.

13 Le Chipko plonge ses racines dans les formes traditionnelles de révolte paysanne, mais en même temps il alimente le discours le plus actuel de l'écologie militante. Notons brièvement son côté feministe: des femmes qui s'enchaînent aux arbres et les embrassent. Au cours de plusieurs épisodes du mouvement, les femmes entrèrent ouvertement en conflit avec les hommes du village qui voulaient, eux, vendre la forêt pour de l'argent frais. Les femmes sont gardiennes en quelque sorte du lien traditionnel qui rend la forêt, source d'eau, de fourrage et de combustible, indispensable à la survie du village $^{20}$. Une idée reçue, propagée par les conférences et les publications de Sunderlal Bahuguna, l'un des protagonistes, fait du Chipko un mouvement quasi romantique de retour à la nature ${ }^{21}$. Il faudrait certes nuancer en analysant - T. Weber et R. Guha sont l'un et l'autre excellents sur ce point - les rapports du Chipko avec le Gandhisme, puis les polémiques au sein du mouvement entre les réalistes (comme C.P. Bhatt à la recherche de technologies de substitution) et les idéalistes qui condamnent la foresterie dans son principe à l'instar de S. Bahuguna déclarant que l'homme, dans la société industrielle, est le boucher de la nature. Cette idéologie traditionnaliste imprègne le Chipko dès l'origine et transforme ce qui n'aurait été autrement rien de plus qu'une révolte paysanne en un mouvement écologiste.

Pour nous, historiens et anthropologues, ce qui justifie la place accordée au Chipko, c'est précisément la continuité qui se manifeste à son propos entre les jacqueries d'autrefois et l'écologie militante d'aujourd'hui. Dans l'histoire des révoltes paysannes au Kumuan et au Garhwal, la non-violence est un trait dominant jusqu'au $x^{\mathrm{e}}$ siècle. Au temps de la royauté, les protestations n'étaient jamais dirigées contre le roi lui-même ; si les choses allaient mal dans le royaume, c'était, supposait-on, que le roi était trompé et desservi par de mauvais ministres; les rebelles ne confondaient pas la monarchie avec l'auteur de leurs maux ${ }^{22}$. Les droits d'usage des villageois faisaient partie intégrante de l'organisation du royaume au même titre que les tenures foncières. Nous pourrions ici encore relativiser l'exemple des forêts himalayennes en faisant référence aux travaux d'indianisme classique qui ont mis en lumière le rapport de la forêt au pouvoir royal ${ }^{23}$. La prospérité du royaume réclame des frontières sûres, et c'est pourquoi les forêts sont des terres domaniales. La frontière par excellence, c'est la forêt dense, la forêt à éléphants dans les textes sanskrits de politique, et le roi possède symboliquement le monopole des éléphants comme celui de la guerre et de la chasse. Les jacqueries ne mettaient pas en question cette idéologie. 

capital. Les révoltes dirigées contre l'exploitation commerciale de ce capital, qui dépossédait les paysans de leurs droits d'usage, ont pris la forme d'incendies volontaires. L'intention était en un premier temps d'enfreindre les lois nouvelles qui interdisaient l'essartage, mais à partir des années 1910, avec des pointes dans la violence comme en 1921, il s'agit bel et bien de détruire la forêt par l'incendie. militants gandhistes, a rompu avec ces formes de révolte destructrice et renoué les liens du paysan à la forêt. Les théoriciens du mouvement s'inspirent de l'un des stéréotypes fondamentaux de l'hindouisme - la forêt source de vie - pour promouvoir l'idée d'une réappropriation de l'environnement. Le dossier ainsi rassemblé sur la forêt dans l'Inde déborde le cadre régional et justifie, me semble-t-il, l'intérêt que suscitent les mouvements écologistes indiens dans la communauté internationale.

\section{NOTES}

1. The State of India's Environment 1982. A Citizen's Report, New Delhi, Centre for Science and Environment 1982, Nelle édition 1985, trad. partielle publiée chez L'Harmattan ; Buddhadeb CHAUDHURI, «Forest, forest-dwellers and forest development », dans B. CHAUDHURI et Asok Kumar MAITI eds., Forest and Forest Development in India, New Delhi, Inter-India Publ., 1989, pp. 19-33 ; Robert REPETTO, « La déforestation des pays tropicaux », Pour la science, 152, Juin 1990, pp. 36-42.

2. George B. SCHALLER, The Deer and the Tiger. A Study of Wildlife in India, Chicago, Univ. of Chicago Press, 1967 ; Francis ZIMMERMANN, La Jungle et le fumet des viandes, Paris, Editions de l'EHESS, Gallimard, Le Seuil, 1982.

3. Marc NICHTER, "Kyasanur Forest Disease : an Ethnography of a Disease of Development ", Medical Anthropology Quarterly, n.s., 1, n 4, 1987, pp. 406-423.

4. Berthold RIBBENTROP, Forestry in British India (1900), New Delhi, Indus Publ., 1989, p. 99 ; Ramachandra GUHA, The Unquiet Woods. Ecological Change and Peasant Resistance in the Himalaya, Delhi. Oxford Univ. Press, 1989. p. 38.

5. Robert CHAMBERS, N.C. SAXENA, Tushaar SHAH, To the Hands of the Poor, Water and Trees, New Delhi, Oxford and IBH Publ., 1989. p. 144.

6. En hindi, « mouvement populaire » (andolan) où les arbres sont « étreints » (chipko).

7. On fait paitre le bétail en forêt. La coutume est d'incendier chaque année le sous-bois pour obtenir une récolte d'herbe, mais le fourrage se compose le plus souvent de feuilles, de glands et de pousses.

8. Vandana SHIVA, Staying alive. Women, Ecology and Survival in India, New Delhi. Kali for Women. 1988, p. 79.

9. Friches traduit imparfaitement le concept de waste lands. Ce sont des terres non arpentées, associées à la vie quotidienne des villageois qui ont le droit d'y faire paître le bétail et d'y prendre bois de chauffage et bois de charpente.

10. R. GUHA, op. cit., pp. 44-46.

11. R. GUHA, op. cit., pp. 51, 59, 139. 
12. Deepak KUMAR (NISTADS. New Dehli). «Problems in Science Administration. A Study of the Survey Organisations in British India 1858-1900». Communication au Colloque. "Sciences et Empires ", Paris, avril 1990, à paraître.

13. R. GUHA, op. cit., pp. 16-20, 129.

14. Jean-Claude GALEY, «Le créancier, le roi, la mort. Essai sur les relations de dépendance dans le Tehri-Garhwal (Himalaya indien) », Purusartha, 4. 1980, pp. 93-163.

15. David LUDDEN, Peasant History in South India. Princeton, Princeton Univ. Press, 1985, p. 123.

16. Eric MEYER, «L'impact de l'économie de plantation sur les structures villageoises ceylanaises : une révolution écologique ? ", in C. COQUERY-VIDROVITCH ed., Sociétés paysannes du tiers-monde, Lille, P.U.L., 1981, pp.171-184: «Bourgeoisie et société rurale au Sri Lanka (1880-1940) », Purusartha, 6, 1982, pp. 223-250.

17. Dès le début des années 1870 , dans le district de Kegalle qu'étudie plus particulièrement $\mathrm{E}$. Meyer, l'État accapare une moitié de ces hautes terres pour les vendre à des planteurs britanniques: les villageois seront acculés à leur vendre l'autre moitié par l'intermédiaire de spéculateurs. La triade indienne des accapareurs que dénonce la bourgeoisie de Colombo en faisant vibrer la fibre xénophobe (Cf E. MEYER. «Bourgeoisie et société rurale..., p. 243)-le Chettiar (l'usurier), le Coolie, auxiliaire fidèle du planteur, et le Kangani (contremâitre indien sur les plantations, qui s'enrichit comme chasseur de terres) - fait écho à la triade Sarkar, Sahukar, Zamindar mentionnée plus haut.

18. B. CHAUDHURI, op. cit., p. 21 : R. CHAMBERS et alii, op. cit., pp. 198 sq.

19. R. CHAMBERS et alii, op. cit., pp. 197-226.

20. Vandana SHIVA, op. cit., R. GUHA,op. cit., p. 173.

21. Thomas WEBER, Hugging the Trees. The Story of the Chipko movement, New Dehli. Viking, 1988/ Penguin Books. 1989, p. 75 ; R. GUHA, op. cit., p. 173.

22. R. GUHA. op. cit., pp. 67, 90, 93. L'auteur fait un rapprochement (p. 187) entre les révoltes qu'il décrit pour la défense des droits d'usage sur la forêt himalayenne et la Guerre dite des Demoiselles, qui fit rage pour les mêmes motifs dans l'Ariège au XIX ${ }^{\mathrm{e}}$ siècle... et on peut lire dans A. CORVOL. L'Homme aux bois.... p. 207, que les Demoiselles ariégeoises criaient parfois «Vive le Roi !».

23. Charles MALAMOUD. "Village et forêt dans l'idéologie de l'Inde brahmanique ", Archives européennes de sociologie, XVII. $\mathrm{n}^{\circ}$ 1. 1976. pp. 3-20 ou Cuire le bois. Rite et pensée dans l'Inde ancienne. Paris. La Découverte. 1989. ch. 4 : Thomas R. TRAUTMANN. «Elephants and the Mauryas » in S.N. MUKHERJEE, ed., India: History and Thought. Essays in honour of A. L. BASHAM. Calcutta. Subarnarckha Publ., 1982. pp. 254-281 : F. ZIMMERMANN, op. cit.

\section{AUTEUR}

\section{FRANCIS ZIMMERMANN}

Francis ZIMMERMANN est directeur de recherche au Centre d'Études de l'Inde et de l'Asie du Sud (EHESS). 